\title{
Wahrung des Besitzstands?
}

Seit den Wahlen 2004 hat der Zentralvorstand nicht nur eine Neuverteilung der Dossiers vorgenommen, sondern der FMH auch neue Strukturen verliehen, die uns dynamischere Überlegungen und raschere Entscheidungsprozesse ermöglichen.

Für eine Beurteilung der langfristigen Auswirkungen ist es zwar noch zu früh, doch die ersten Ergebnisse dieser Neuerungen sind ermutigend. Die Strukturreform ist allerdings noch nicht abgeschlossen: Zurzeit werden neue Führungsmodelle für den ZV geprüft, die der Ärztekammer im Dezember 2007 vorgelegt werden.

Angesichts der Herausforderungen und Entwicklungen, die in den nächsten zehn Jahren in der Gesundheitspolitik anstehen, ist diese Flexibilität unerlässlich. Die bevorstehenden Reformen können von der Leitung der FMH nicht vorgeschrieben werden, sondern müssen von der Mehrheit der Ärzteschaft unterstützt werden. Dies scheint heute nicht gewährleistet zu sein. Oft ist es schwierig, Verständnis für Reformen zu wecken, die auf eine Neupositionierung der Ärzteschaft in der Gesundheitspolitik ausgerichtet sind, da wir uns noch zu stark von einer Politik der Besitzstandswahrung leiten lassen.

Im Zentrum unserer ärztlichen Tätigkeit wird weiterhin die einzigartige Beziehung zwischen dem Arzt und seinen Patienten stehen. Im Rahmen dieser Beziehung müssen wir die vertrauliche Behandlung der Anfragen, die an uns gerichtet werden, die Freiheit der diagnostischen Abklärung sowie die Wahl der Behandlung und der Präventionsmassnahmen wahren. Dieser bisher unangefochtene Kern steht heute mehr und mehr unter Druck. Der Druck rührt von den Wirksamkeits-, Wirtschaftlichkeits- und Effizienzkriterien her, die uns zu Recht auferlegt werden, aber auch von unseren «klassischen» Idealen in bezug auf die Patientenversorgung. Daher ist dieser Druck vielleicht weniger auf «Aggressoren» zurückzuführen, die es auf die Ärzteschaft abgesehen haben. Vielmehr entsteht er aus einer zunehmenden Komplexität der Wechselbeziehungen zwischen den Akteuren des Gesundheitswesens einerseits und den Anforderungen einer verunsicherten und immer anspruchsvolleren Gesellschaft andererseits. In diesem Umfeld haben wir alle ein Interesse daran, uns für die Einzigartigkeit dieser Beziehung in einem System einzusetzen, das einen Zugang zu einer hochwertigen medizinischen Versorgung $\mathrm{zu}$ realistischen Kosten gewährleistet.

In den kommenden Jahren zeichnen sich zahlreiche Herausforderungen ab, wie zum Beispiel 2008 das Auslaufen des Zulassungsstopps für neue Arztpraxen. Das derzeitige Modell führt zu einer Überfüllung der Spitäler mit jungen Ärztinnen und Ärzten. Es ermöglicht jedoch auch - was derzeit noch ein Tabuthema ist - eine Regu- lierung der Ärztedichte in bestimmten Städten und Kantonen. Diesbezüglich wird Gesundheitsminister Pascal Couchepin nächstens an uns gelangen und unsere Vorschläge anhören. Hier wird es darum gehen, unsere politische Verantwortung wahrzunehmen, was selbstverständlich eine Antwort vom Typ «Status quo» ausschliesst.

Die Aufhebung des Kontrahierungszwangs ist eine weitere hängige Frage. Bisher war unser Standpunkt zur Aufhebung dieser Klausel stets ein klares und bedingungsloses Nein. Ist diese Haltung heute immer noch optimal? Wir müssen sie unbedingt unter Berücksichtigung der derzeitigen Probleme des Gesundheitswesens und der demographischen Entwicklung der Ärzteschaft beurteilen. Eine mögliche Antwort darauf könnte zum Beispiel eine bessere Vernetzung unserer Tätigkeiten sein.

Und was ist zum Tarifsystem TARMED zu sagen? Da es offensichtlich nicht vollständig befriedigt, soll es korrigiert und angepasst werden. Werden wir zuwarten, bis andere Akteure des Gesundheitswesens dies für uns tun? Hier bieten die LeiKoV und SwissDRG erste Lösungsansätze. Allerdings erscheint es wenig realistisch, die DRG auf die stets einzigartige Klinik im ambulanten Bereich anzuwenden.

Welche Standpunkte werden wir schliesslich im Bereich der Ausbildung vertreten, die für die Qualität unserer Arbeit, aber auch für die Attraktivität unseres Berufs verantwortlich ist? Soll für die ersten Anfragen unserer Patienten die Idee einer sechsjährigen Ausbildung der Ärztinnen und Ärzte unterstützt werden? Oder würden im Gegenteil drei Jahre reichen? Auf diese Fragen müssen unbedingt nuancierte, wohlbedachte Antworten gefunden werden.

Eines erscheint sicher: In den erwähnten Beispielen ist die Politik der Besitzstandswahrung fehl am Platz. Vor allem ermöglicht sie uns nicht, gegenüber unseren Partnern, von denen wir ebenfalls verlangen, dass sie ihre oft festgefahrenen Positionen aufgeben, einen verantwortungsbewussten klinischen und politischen Standpunkt einzunehmen. Wir müssen daher innovativ sein und partnerschaftlich neue Modelle vorschlagen. Diese sollen der Bevölkerung die für die Qualität notwendige Vielfalt beim Zugang zur medizinischen Versorgung und die Vertraulichkeit der Beziehung zwischen Arzt und Patient gewährleisten, aber auch die wissenschaftliche und wirtschaftliche Attraktivität dieses wunderbaren Berufs sicherstellen.

\section{Dr. med. Jacques de Haller,} Präsident der FMH

Dr. med. R. Raggenbass, Psychiater und Psychotherapeut FMH, Mitglied des Zentralvorstands der FMH, Leiter des Ressorts Gesundheit und Prävention 